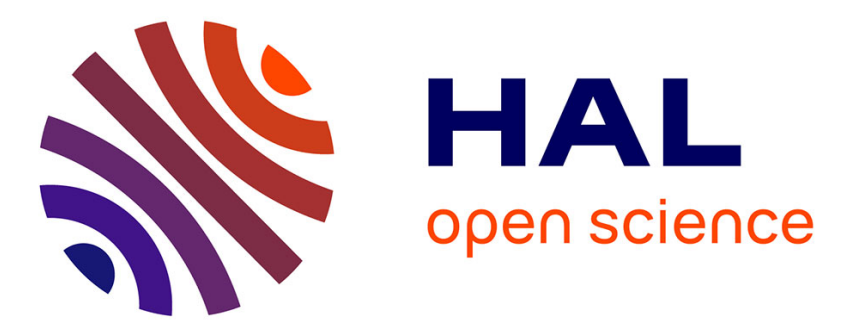

\title{
Sound velocity and equation of state of liquid Cesium at high pressure and high temperature
}

Frederic Decremps, Simon Ayrinhac, Michel Gauthier, Daniele Antonangeli, Marc Morand, Yiuri Garino, Paraskevas Parisiades

\section{- To cite this version:}

Frederic Decremps, Simon Ayrinhac, Michel Gauthier, Daniele Antonangeli, Marc Morand, et al.. Sound velocity and equation of state of liquid Cesium at high pressure and high temperature. Physical Review B: Condensed Matter and Materials Physics (1998-2015), 2018, 98 (18), 10.1103/PhysRevB.98.184103 . hal-01920237

\section{HAL Id: hal-01920237 https://hal.sorbonne-universite.fr/hal-01920237}

Submitted on 13 Nov 2018

HAL is a multi-disciplinary open access archive for the deposit and dissemination of scientific research documents, whether they are published or not. The documents may come from teaching and research institutions in France or abroad, or from public or private research centers.
L'archive ouverte pluridisciplinaire HAL, est destinée au dépôt et à la diffusion de documents scientifiques de niveau recherche, publiés ou non, émanant des établissements d'enseignement et de recherche français ou étrangers, des laboratoires publics ou privés. 


\title{
Sound velocity and equation of state of liquid Cesium at high pressure and high temperature
}

\author{
Frédéric Decremps, ${ }^{*}$ Simon Ayrinhac, Michel Gauthier, Daniele Antonangeli, Marc Morand, \\ Yiuri Garino, and Paraskevas Parisiades \\ Sorbonne Université - CNRS - Muséum National d'Histoire Naturelle, \\ Institut de Minéralogie de Physique des Matériaux et de Cosmochimie, 4 Place Jussieu, F-75005 Paris, France
}

(Dated: November 4, 2018)

\begin{abstract}
Liquid cesium (l-Cs) sound velocity at high densities was investigated along a $500 \mathrm{~K}$ isotherm using high-pressure picosecond acoustics measurements. At $2.0 \mathrm{GPa}$, the liquid sound velocity goes through a maximum versus pressure without any change on the reflectivity and interferometry acoustic signals. Upon further compression, a softening of the l-Cs visco-elastic properties is observed from 2.0 up to $4.0 \mathrm{GPa}$, pressure at which the reflectometric signal is abruptly reversed whereas the interferometric signal remains qualitatively the same. This anomalous behaviour could be related to an electronic transformation within the l-Cs state, which here again could reflect what happens at lower temperature within the solid state. If so, such liquid-liquid transition may be driven by the progressive collapse of the $6 s$ electronic orbital onto the $5 d$ ones. Above $4.0 \mathrm{GPa}$, the l-Cs sound velocity starts again to increase as commonly expected upon compression.
\end{abstract}

PACS numbers: 62.50.+p, 62.60.+v, 47.35.Rs, 65.40.De, 61.10.Nz, 61.20.-p, 61.25.Mv

\section{INTRODUCTION}

Alkali metals are chemically considered as archetypal examples of simple liquids at ambient pressure $^{1}$. At higher densities, they however depart from their single free-electron behavior, showing a sequence of symmetrybreaking transitions which is well known to exhibit richness $^{2,3}$ (many phases), complexity ${ }^{4-7}$ (low-symmetry structures, collective excitations), and puzzling mechanism $^{8,9}$ (driven by core electrons or zero-point contribution). Recently, the melting curves and the equation of state of some of the group-I elements have also been determined over a large pressure and temperature ranges, showing unexpected behaviors ${ }^{10,11}$. However, both experimental and theoretical difficulties still prevent extensive studies of their fluid states under high pressure. Among the many hardships, we can mention the existence of diffusion and relaxation processes within alkali elements, or their high chemical reactivity with consequent complications in the containment under extreme conditions, which result in a quite limited bibliography.

Solid cesium has been pioneeringly studied by Bridg$\operatorname{man}^{12}$, who discovered two consecutive phase transitions nearly at $2.3 \mathrm{GPa}$ and $4.5 \mathrm{GPa}$. At $300 \mathrm{~K}$, Cs is bodycentered cubic (bcc, Cs-I), stable up to $2.3 \mathrm{GPa}$, where it transforms to the face-centered cubic (fcc) Cs-II phase. An orthorhombic Cs-III exists over a very narrow pressure range ${ }^{3}$ (between 4.2 and $4.3 \mathrm{GPa}$ ), which then transforms to the non-close-packed tetragonal Cs-IV (stable up to $12 \mathrm{GPa}$ ). While the bcc-to-fcc phase transition is purely structural, the transformation around $4.2 \mathrm{GPa}$ has an electronic character ${ }^{13,14}$, where conduction electrons are transferred from the $6 s$ to the $5 d$ band.

The high pressure behavior of solid Cs has been largely studied over the years as this element is one of the most compressible substances over the whole alkali family (at $10 \mathrm{GPa}$, it is compressed to about one third of its volume at ambient pressure ${ }^{15}$ ). As a consequence, Cs is advantageously expected to experience at relatively low pressure the same effects other less compressible monoatomic liquid metals undergo at much higher pressure, as lithium ${ }^{10}$. This hypothesis is validated through many experimental observations, as the existence of a bcc-to-fcc phase transition, of common complex structures (i.e. the orthorhombic one), or as the presence of a sequence of maxima and minima in the melting curve. In the liquid state, and mainly because of the technical difficulties mentioned above, only a couple of studies have been published on Cs. In the study of Tsuji et al. ${ }^{16}$, the pressure evolution of l-Cs has been followed through the modification of the structure factor $\mathrm{S}(\mathrm{Q})$. They observed changes on both the shape and the intensity of $\mathrm{S}(\mathrm{Q})$, suggesting the existence of two liquid-liquid transitions (LLT). A first transformation was seen to occur at $2.0 \mathrm{GPa}$, while a second one is suggested between 3.0 and $4.3 \mathrm{GPa}$, pressure range where a slender decrease of the $\mathrm{S}(\mathrm{Q})$ intensity is observed. However, such exciting conclusions have been derived on the basis of only four experimental data points, all collected at different temperatures. In addition, the measurement of S(Q) was experimentally limited to about $7 \AA^{-1}$, which hinder a reliable extraction of the pair distribution $g(r)$, coordination number $C_{N N}$, or of the atomic density $\rho$ from experimental data. Subsequent more advanced $\mathrm{x}-$ ray diffraction measurements by Falconi et al. ${ }^{17}$ came to different conclusions. Here, many data points have been recorded along two different isotherms, at 493 and 623 $\mathrm{K}$. The main outcome of this work is the occurrence of only one single LLT, at $3.9 \mathrm{GPa}$, characterized by a very large density jump (17\%), and a coordination number going down from 12 to 8 . These results are also supported by ab initio simulations performed one year later by the same group ${ }^{18}$. The common underlying hypothesis of these works is a close similarity between the behavior of the liquid and what observed within the crystalline state, 
say the existence of $d s p^{3}$ electronic hybridization. However, the most recent experimental work carried out by $\mathrm{x}$-ray absorption (see Hattori et al..$^{19}$ ) do not support any abrupt density jump at least up to $5 \mathrm{GPa}$ and argues for a progressive $6 s-5 d$ electrons transfer.

\section{EXPERIMENTAL METHODS}

Experimentally, combined picosecond ultrasonics technique and diamond anvils cell (DAC) ${ }^{20}$ has been proven to overcome many of previous technical barriers, and now enable to study subtle changes in liquid metals properties at high densities ${ }^{21}$. Using such state-of-the-art approach, we have here determined the pressure evolution along a $500 \mathrm{~K}$ isotherm of the l-Cs sound velocity with precision better than $1 \%$. High temperature was generated through the use of a resistive external heater surrounding the whole DAC. The temperature was regulated using an electronic module and measured with an accuracy better than $1 \mathrm{~K}$ by two K-type thermocouples glued on each anvil. Using a glove box, ultra-pure Cs from Sigma Aldrich $(99.99 \%)$ was loaded fully embedded into a rhenium gasket hole of $250 \mu \mathrm{m}$ diameter placed in a DAC equipped with $600 \mu \mathrm{m}$ culet size diamonds.

Our acoustic set-up, described in reference $\left[{ }^{22}\right]$, is based on the use of an ultrashort pulse of 60 fs generated every $12.554 \mathrm{~ns}$ by a Ti:Sapphire laser operating at $\lambda=800 \mathrm{~nm}$. The laser beam is split into pump and probe beams. The pump is focused on one surface of the sample (focused spot of about $3 \mu \mathrm{m}$ ) through a diamond window and absorbed in a length scale $\xi(\approx 36 \mathrm{~nm})$ whereas the probe is focused on the opposite side through the other diamond window. As soon as the pump laser pulse reaches the surface, it creates a sudden and small temperature rise $\Delta T$ (less than $10 \mathrm{~K}$ ) which relaxes by launching an acoustic strain field (with frequency typically around the $\mathrm{GHz}$ in a metallic liquids) propagating at the sound velocity $v_{l}$.

In a pure thermo-elastic model ${ }^{23,24}$, the time and space reflectance change $\delta r(t)$ of the probe is a function of the photo-elastic coefficient $\partial \tilde{n} / \partial \eta$ (where $\tilde{n}$ is the sample refractive index, and $\eta$ the stress generated by thermal expansion) and the surface displacement along the propagation axis $z\left(u_{0}(t)=\int_{0}^{+\infty} \eta(z, t) d z\right)$ :

$$
\begin{aligned}
\frac{\delta r(t)}{r_{0}} & =2 i k_{0} n_{d}\left\{\int_{0}^{+\infty} \eta(z, t) d z\right. \\
& \left.+\left(\frac{\partial \tilde{n}}{\partial \eta}\right) \frac{2 \tilde{n}}{n_{d}^{2}-\tilde{n}^{2}} \int_{0}^{+\infty} \eta(z, t) e^{2 i k_{0} \tilde{n} z} d z\right\}
\end{aligned}
$$

where $k_{0}$ is the laser wave vector and $n_{d}$ the refractive index of diamond.

To summarize, after propagation across the sample both thermal and acoustic effects alter the optical reflectivity of the sample in two ways : the photo-elastic effect and the surface displacement (as the acoustic echo reaches the surface). The first modification contributes to the change of the intensity of the reflectivity and its phase shift, whereas the second one only modifies the phase of the reflectivity signal.

We performed two different runs with two different sample loadings. In both cases, pressure was increased to the maximum value (5 GPa) and data have been collected downstroke. The temporal method (described in a previous article ${ }^{22}$ ) enables to extract the travel time $\Delta t$ corresponding to a single way of the acoustic wave into l-Cs. Sound velocity was then determined through the measurement of $\Delta t$ during decreasing pressure at continuous rate of the order of $-0.02 \mathrm{bar} / \mathrm{min}$ on the membrane DAC. Pressures were determined from the $\mathrm{Sr}_{4} \mathrm{O}_{7}: \mathrm{Sm}^{2+}$ fluorescence line shift before and after collection of each acoustic scope (pressure uncertainty given by symbols sizes in all figures). Using the imagery method ${ }^{22}$, the gasket thickness was determined simultaneously with the sound velocity at two pressures, $4.8 \mathrm{GPa}$ and $2.3 \mathrm{GPa}$, leading in both cases to a constant value of $e=20(2) \mu m$. Moreover, by white light interferences, we also estimated the thickness of the recovered gasket at ambient conditions to be 21(1) $\mu \mathrm{m}$. A constant thickness upon decompression is consistent with many other experimental observations $^{22,25,26}$ and has been explained through the study of plasticity processes inside the rhenium gasket ${ }^{27}$ under relaxing stresses.

\section{RESULTS AND DATA ANALYSIS}

The sound velocity determined from our measurements of travel time as a function of pressure is in agreement with an extrapolation of the low pressure ultrasonic data from Shaw ${ }^{28}$.

Fig. 1 shows the pressure evolution of of the l-Cs sound velocity at a constant temperature of $500 \mathrm{~K}$. The linear dependence, expected for simple metals, only stands up to about $0.3 \mathrm{GPa}$. The low frequency limit of the sound velocity measured using inelastic $\mathrm{x}$-ray scattering at $1 \mathrm{GPa}$ and $493 \mathrm{~K}\left(v=1.18 \mathrm{~km} \cdot \mathrm{s}^{-1}\right.$, see $\left.\mathrm{Ref}^{5}\right)$ is somewhat lower but in overall agreement with present adiabatic sound velocity. At higher pressures, the behavior becomes highly non-linear, with a flattening and a maximum at $2.0 \mathrm{GPa}$ (pressure value in the vicinity of the bcc-fcc transition in the solid), but without discontinuity in the sound speed.

A further anomaly is observed between 2 and $4 \mathrm{GPa}$, where the velocity of the longitudinal wave decreases with pressure. Interestingly, this quite unusual behavior occurs over a pressure range where the liquid is denser than the solid ${ }^{13}$. Likewise, but to a lesser extent, we here emphasize that this softening was also present in the solid Cs-II phase between $\sim 3.5 \mathrm{GPa}$ and $4.2 \mathrm{GPa}$, as reported by Voronov et al. ${ }^{29}$ (see Fig. 1). This peculiar evolution is thermodynamically authorized, and can be here understood taking into account the existence of an hybridization between $s$ and $d$ orbitals. In a same manner, pure $\mathrm{Ce}$ is another well known example ${ }^{30,31}$, where the 


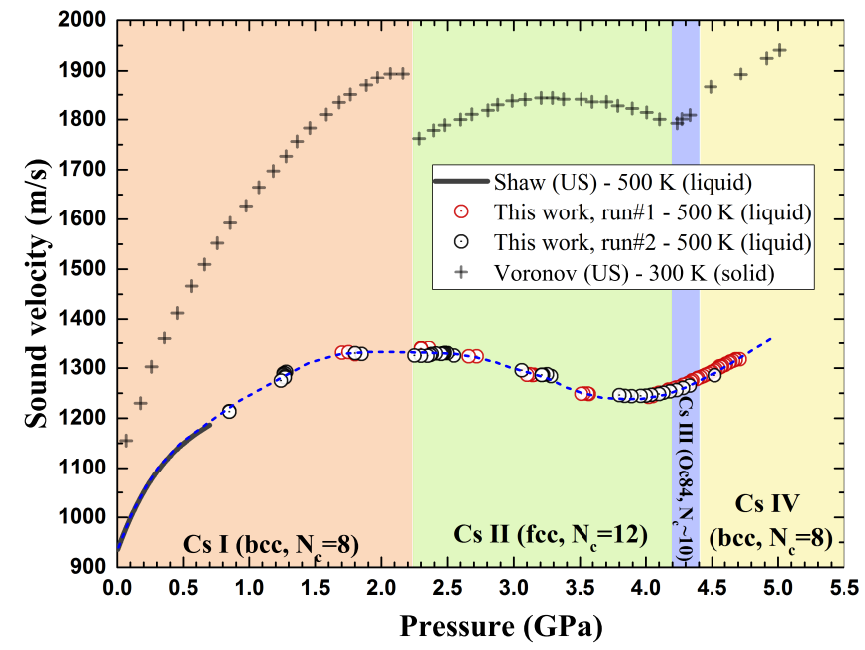

FIG. 1. (color online) Longitudinal wave velocities of l-Cs as a function of pressure for the present work $(\mathrm{T}=500 \mathrm{~K}$, two runs, black and red open circles; dotted line, as guides for the eyes), plotted along with low-pressure ultrasonic data on l-Cs at $500 \mathrm{~K}$ from $\operatorname{Ref}^{28}$ (black line) and ultrasonic measurements on solid-Cs at $300 \mathrm{~K}$ from $\operatorname{Ref}^{29}$ (crosses). The maximal uncertainties associated with the relative sound velocity $\Delta v_{l} / v_{l}$ is less than $0.5 \%^{22,26}$.

$4 f$-electron delocalization is at the origin of the reduction of the bulk modulus with pressure, an anomaly which is also observed well prior to the phase transition. In the present case, a progressive $s$ - $d$ hybridization in Cs is thus likely to be at the origin of the observed elastic softening occurring up to $4.0 \mathrm{GPa}$, pressure at which the sound velocity starts again to increase. Here again, similar behavior is observed in the solid Cs phase ${ }^{29}$, at the same pressure range where a structural phase transformation occurs in the solid phase below $500 \mathrm{~K}$.

In order to discuss the electronic nature of the two consecutive extrema (the maximum at $2.0 \mathrm{GPa}$, and the minimum at $4.0 \mathrm{GPa}$ ), we can exploit the complementary of the two detection methods.

In both interferometric and reflectometric set-up, the shape of the strain depends on the acoustic impedance of the diamond anvils $Z_{d}=\rho v_{l}$ and the sample $Z$ (where $\rho$ is the density and $v_{l}$ the longitudinal sound velocity) via the acoustic strain reflection coefficient $r_{a c}=\left(Z_{d}-\right.$ $Z) /\left(Z_{d}+Z\right)$. In the present case $r_{a c}$ is large and positive $(\approx 0.85)$, leading to a unipolar strain pulse shape in a simple thermoelastic generation theory. Moreover, since the laser probe is absorbed on a small $\xi$ range, equation 1 becomes:

$$
\begin{aligned}
\frac{\delta r(t)}{r_{0}} & \approx 2 i k_{0} n_{d}\left\{\left(e^{-v_{l} t / \xi}-\left(1-r_{a c}\right)\right) \xi \eta_{0} / 2\right. \\
& \left.+\left(\frac{\partial \tilde{n}}{\partial \eta}\right) \frac{2 \tilde{n}}{n_{d}^{2}-\tilde{n}^{2}} v_{l} t e^{-v_{l} t / \xi} \eta_{0} / 2\right\}
\end{aligned}
$$

The interferometric configuration allows the determi- nation of the imaginary part of the reflectivity change:

$$
\begin{gathered}
\Im\left(\frac{\delta r(t)}{r_{0}}\right) \approx 4 k_{0} n_{d} \frac{u_{0}(t)}{2}+4 k_{0} n_{d} v_{l} t \eta(0, t) \times \\
\frac{\left(\frac{\partial n}{\partial \eta} n-\frac{\partial \kappa}{\partial \eta} \kappa\right)\left(n_{d}^{2}-\left(n^{2}-\kappa^{2}\right)\right)-\left(\frac{\partial n}{\partial \eta} \kappa+\frac{\partial \kappa}{\partial \eta} n\right) 2 n \kappa}{\left(n_{d}^{2}-\left(n^{2}-\kappa^{2}\right)\right)^{2}+(2 n \kappa)^{2}}
\end{gathered}
$$

We here emphasize that the change in phase of $\frac{\Delta r(t)}{r_{0}}$
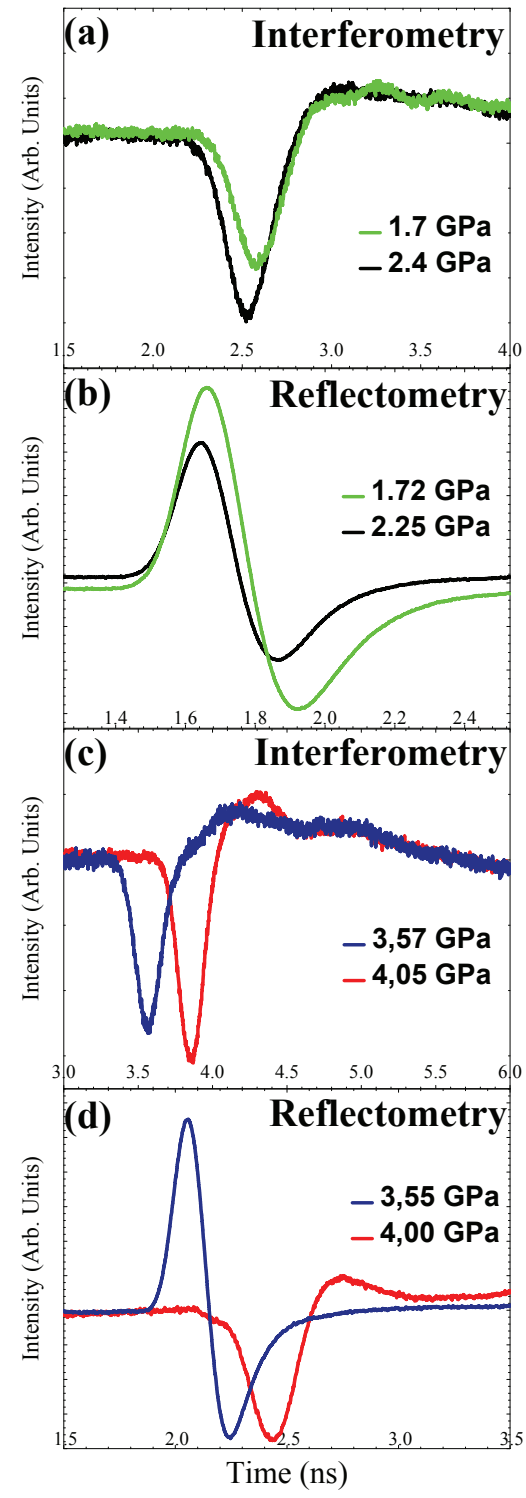

FIG. 2. (color online) Reflectivity change of l-Cs at $500 \mathrm{~K}$ as a function of the optical probe-pulse time delay. The modification of the optical reflectivity due to the acoustic strain has been recorded around $2 \mathrm{GPa}$ using two types of detection, reflectometry (a) and interferometry (b). Same but around 4 $\mathrm{GPa}$ are given in (c) and (d) respectively. The pulse shape qualitatively varies (shifted in phase by about $\pi$ ) only in the vicinity of $4 \mathrm{GPa}$ and using a reflectometric detection. 
(of about $10^{-7}$ ) is generally assumed to be mainly due to a surface displacement $u_{0}(t)$ (as small as $10 \mathrm{fm}$ ).

Within the reflectometry configuration, the variation of reflectivity as a function of time is detected through the direct measurement of the intensity modification of the probe. The signal is thus given by the real part of equation 2 as:

$$
\begin{array}{r}
\Re\left(\frac{\delta r(t)}{r_{0}}\right) \approx 4 k_{0} n_{d} v_{l} t \eta(0, t) \times \\
\frac{\left(\frac{\partial n}{\partial \eta} \kappa+\frac{\partial \kappa}{\partial \eta} n\right)\left(n_{d}^{2}-\left(n^{2}-\kappa^{2}\right)\right)+\left(\frac{\partial n}{\partial \eta} n-\frac{\partial \kappa}{\partial \eta} \kappa\right) 2 n \kappa}{\left(n_{d}^{2}-\left(n^{2}-\kappa^{2}\right)\right)^{2}+(2 n \kappa)^{2}}
\end{array}
$$

The pulse profiles corresponding to equations (4) and (3) are shown in Fig. 2 for the two pressure ranges of interest around 2 and $4 \mathrm{GPa}$ respectively. The pulse shape observed by interferometry does not significantly change under pressure, it only shifts. The same behavior is observed on the optical signal measured using the reflectometric configuration at $2.0 \mathrm{GPa}$; on the contrary, it is largely modified around 4.0 GPa (change of sign).

\section{DISCUSSION}

The pressure value of $2.0 \mathrm{GPa}$, where we document a maximum in sound velocity for the l-Cs, closely corresponds to CsI-CsII solid-solid phase transition, known to be purely structural (from eightfold coordinated bcc structure to 12 -coordinated fcc structure ${ }^{2,3}$ ). Furthermore, an analysis of the x-ray diffraction data in l-Cs by Falconi et al. ${ }^{17}$ shows a plateau in the evolution of the coordination number from ambient conditions up to 2.0 GPa (pressure after which $C_{N N}$ begins to decrease), an observation not discussed by the authors but which could be either related to a pre-transitional effect ahead of the density change at $4 \mathrm{GPa}$ (Falconi's point of view) or to the occurrence of a distinct transition pressure at $2 \mathrm{GPa}$. This last assumption would support a structural nature of the anomaly in the liquid state above 2.0 GPa probably related to the fact that (as observed for $1-\mathrm{Rb}^{7}$ ) l-Cs is not a simple liquid anymore and long-range repulsion have to be taken into account.

More complex is the analysis of the data at $4.0 \mathrm{GPa}$ as change in the sign of only reflectivity is related to the relative values of the l-Cs refractive index and its of diamonds anvil. From ambient to low pressures, the refractive index of cesium is weaker than the diamond one, whereas at a moderated pressure due to the low compressibility of diamond and the huge compressibility of cesium the opposite scheme is expected. If the optical absorption of cesium is significant, it would smoothly go down to zero in intensities in both interferometric and reflectivity set-ups. This is clearly not what is here observed, with a sharp phase change of the reflectivity shape.

Nothing in equation 4 can account for a such abrupt change of the reflectivity signal without the same abrupt variation on the optical parameters $n, \kappa$ or on the elastooptical $\partial \kappa / \partial \eta$ and $\partial n / \partial \kappa$. This observation suggests that the change of shape (i.e. of signal phase) at $4.0 \mathrm{GPa}$, concomitant to the second extrema of the $v(P)$ evolution in l-Cs, signs a sudden modification either of the complex optical index or of the complex photo-elastic coefficient (or both) of the sample itself, thus pointing to an electronic nature of the transition.

An hypothesis would be that the electronic-character of a possible liquid-liquid transition observed by the present study finds its twin-image within the solid phase diagram, where a transition around $4.2 \mathrm{GPa}$ is accompanied by abrupt changes in resistivity ${ }^{13}$, phenomenon attributed to the collapse of the $6 s$ electronic orbital onto the $5 d$ orbitals (electron transfer).

Such a subtle transformation within the liquid state should come with smooth discontinuities in the fluid equation of state $V(P)$, difficult to be detected by diffraction. Acoustic measurements are known as a very reliable alternative, since sound velocity is very sensitive to subtle changes in long-range order.

As illustrated in Fig. 3, the evolution of the liquid density with pressure along an isotherm at $500 \mathrm{~K}$ (extracted from the present sound velocity measurements on the basis of exact thermodynamic relations, an approach that have been already proven for many different metallic liquids $^{22,26}$ ) overall well compares with most recent density determination by $\mathrm{x}$-ray absorption technique ${ }^{19}$, at least to the extent that any sharp discontinuity in density is

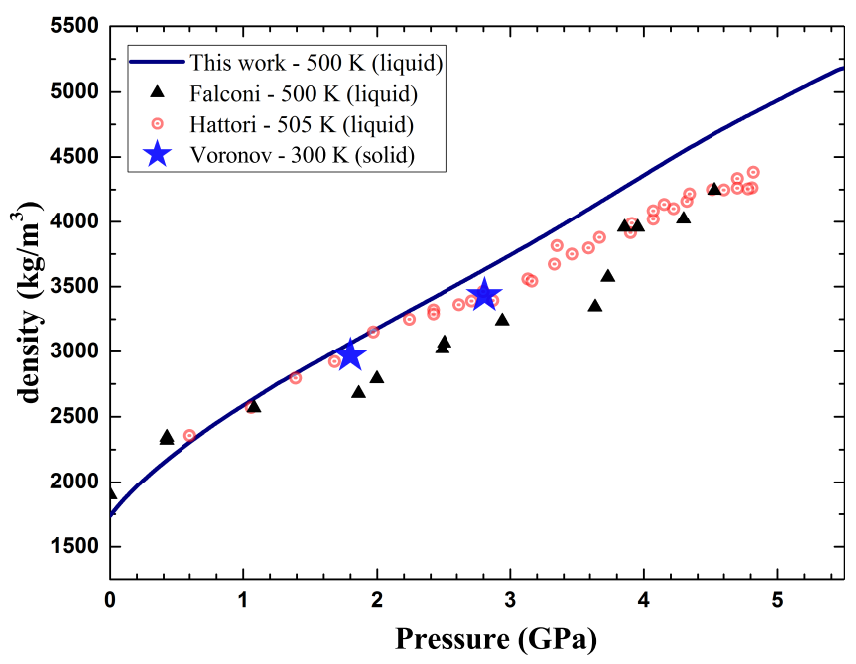

FIG. 3. (color online) Density $(\rho)$ of l-Cs at $500 \mathrm{~K}$ as a function of pressure deduced from present measurements, and compared to previous studies (x-ray diffraction results from Falconi ${ }^{17}$ and $x$-ray absorption from Hattori ${ }^{19}$ ). The maximal uncertainties associated with the relative densities $\Delta \rho / \rho$ is estimated of about $2 \%^{22,26}$. The two black stars correspond to solid densities ${ }^{29}$ at the pressure where two maxima is observed on the melting line, where both solid and liquid phases have the same density. We here emphasize the clear agreement between our data and those from Hattori ${ }^{19}$. 
observed for increasing pressure, note even across liquidliquid transitions. Fig. 3 clearly emphasizes that a huge anomaly on the pressure dependence of the sound velocity does not necessarily comes with a clear effect on the equation of state. Indeed, while the pressure dependence of the volume is associated to the first derivative of the internal energy, the sound speed vs pressure is directly related to the second derivative with respect to the strain.

\section{CONCLUSION}

In summary, picosecond acoustics measurements on lCs under extreme conditions have provided experimental evidence of an S-shaped pressure dependence of sound velocity, with a maximum at $2.0 \mathrm{GPa}$ and a minimum at $4.0 \mathrm{GPa}$. The first singularity could be related to a local change of the liquid structure, whereas present data do not provide a conclusive evidence for such behavior. The second anomaly (minimum at $4 \mathrm{GPa}$ ) is likely correlated to modifications of the electronic properties, as argued from the optical reflectivity measurements. By and large, the occurrence of such behavior within the liquid phase, never observed up to now, shows that under pressure l-Cs may mimic its solid phase, though at lower corresponding densities, as expected by the quasicrystalline paradigm, speculation which has to be tested using further simulation or experimental data.
* frederic.decremps@sorbonne-universite.fr

1 T. S. Ingebrigtsen, T. B. Schrøder, and J. C. Dyre, Phys. Rev. X 2, 011011 (2012).

2 H. Katzke and P. Tolédano, Phys. Rev. B 71, 184101 (2005).

3 M. I. McMahon, R. J. Nelmes, and S. Rekhi, Phys. Rev. Lett. 87, 255502 (2001).

4 V. F. Degtyareva, Solid State Sciences 36, 62 (2014).

5 V. M. Giordano and G. Monaco, J. Chem. Phys. 131, 014501 (2009).

${ }^{6}$ T. Bryk, S. De Panfilis, F. A. Gorelli, E. Gregoryanz, M. Krisch, G. Ruocco, M. Santoro, T. Scopigno, and A. P. Seitsonen, Phys. Rev. Lett. 111, 077801 (2013).

7 F. A. Gorelli, S. De Panfilis, T. Bryk, L. Ulivi, G. Garbarino, P. Parisiades, and M. Santoro, J. Phys. Chem. Lett. 9, 2909 (2018).

8 N. E. Christensen and D. L. Novikov, Phys. Rev. Lett. 86, 1861 (2001).

9 A. F. Goncharov, M. Gauthier, D. Antonangeli, S. Ayrinhac, F. Decremps, M. Morand, A. Grechnev, S. M. Tretyak, and Y. A. Freiman, Phys. Rev. B 95, 214104 (2017).

10 E. Gregoryanz, O. Degtyareva, M. Somayazulu, R. J. Hemley, and H.-k. Mao, Phys. Rev. Lett. 94, 185502 (2005).

11 A. M. J. Schaeffer, W. B. Talmadge, S. R. Temple, and S. Deemyad, Phys. Rev. Lett. 109, 185702 (2012).

12 P. W. Bridgman, Proc. Am. Acad. Arts Sci. 76, 71 (1948).

13 A. Jayaraman, R. C. Newton, and J. M. McDonough, Phys. Rev. 159, 527 (1967).

14 G. C. Kennedy, A. Jayaraman, and R. C. Newton, Phys. Rev. 126, 1363 (1962).

15 K. Takemura and K. Syassen, Phys. Rev. B 32, 2213 (1985).
${ }^{16}$ K. Tsuji, , K. Yaoita, M. Imai, T. Mitamura, T. Kikegawa, O. Shimomura, and H. Endo, Journal of Non-Crystalline Solids 117-118, 72 (1990).

17 S. Falconi, L. F. Lundegaard, C. Hejny, and M. I. McMahon, Phys. Rev. Lett. 94, 125507 (2005).

18 S. Falconi and G. J. Ackland, Phys. Rev. B 73, 184204 (2006).

19 T. Hattori, Phys. Rev. B 97, 100101 (2018).

20 F. Decremps, L. Belliard, B. Perrin, and M. Gauthier, Phys. Rev. Lett. 100, 035502 (2008).

21 F. Decremps, M. Gauthier, S. Ayrinhac, L. E. Bove, L. Belliard, B. Perrin, M. Morand, G. Le Marchand, F. Bergame, and J. Philippe, Ultrasonics 56, 129 (2015).

22 S. Ayrinhac, M. Gauthier, L. E. Bove, M. Morand, G. Le Marchand, F. Bergame, J. Philippe, and F. Decremps, J. Chem. Phys. 140, 244201 (2014).

23 O. Matsuda, M. C. Larciprete, R. Li Voti, and O. B. Wright, Ultrasonics 56, 3 (2015).

24 O. B. Wright, B. Perrin, O. Matsuda, and V. E. Gusev, Phys. Rev. B 78, 024303 (2008).

25 A. Dewaele, J. H. Eggert, P. Loubeyre, and R. Le Toullec, Phys. Rev. B 67, 094112 (2003).

26 S. Ayrinhac, M. Gauthier, G. Le Marchand, M. Morand, F. Bergame, and F. Decremps, Journal of Physics: Condensed Matter 27, 275103 (2015).

27 D. J. Dunstan, Review of Scientific Instruments 60, 3789 (1989).

28 G. H. Shaw and D. A. Caldwell, Phys. Rev. B 32, 7937 (1985).

29 F. Voronov, O. Stalgorova, and E. Gromnitskaya, J. Exp. Theor. Phys. 32, 77 (2002).

30 F. Decremps, D. Antonangeli, B. Amadon, and G. Schmerber, Phys. Rev. B 80, 132103 (2009).

31 D. Antonangeli, D. L. Farber, C. M. Aracne, D. G. Ruddle, J. Siebert, and B. P. Bonner, High Pressure Research 30, 151 (2010). 